\title{
Interactions between a general practitioner and representatives of drug companies
}

\section{Brian O'Mahony}

The Surgery, Lismore, County Waterford, Republic of Ireland Brian O'Mahony, general practitioner

BMf 1993;306: 1649
Nearly all general practitioners meet representatives of drug companies. ${ }^{1}$ The drug industry spent an estimated $£ 5000$ per general practitioner promoting its products in the United Kingdom in 1985.2 Drug representatives are a crucial element in the promotion of products, and expenditure on representatives accounts for over $50 \%$ of promotional budgets. Information is available on the frequency and duration of meetings between general practitioners and drug companies' representatives.' I describe my meetings with representatives during one year, including the samples, gifts, and literature given to me.

\section{Subjects, methods, and results}

The study was based at my singlehanded general practice in a rural town, population 1000, in the south east of Ireland. The practice was not dispensing. For 50 of the 52 weeks between 29 August 1990 and 28 August 1991 I and two receptionists recorded details of the visits by representatives of drug companies to my surgery. We recorded each representative's name and gender, the name of the company represented, the time spent with me, the name and number of products promoted, and any samples, gifts, or literature provided. The representatives were unaware that the study was taking place. For each visit I adopted a passive role, allowing the representative to dictate its content and duration. I did not request any samples but accepted all samples and gifts that were offered. The value of the samples was calculated from the prices quoted for September 1991 in the Monthly Index of Medical Specialities.

I received a total of 109 visits, a mean of $2 \cdot 2$ per week, from 49 representatives of 41 drug companies, a mean number of 2.7 visits per company. The visits lasted for a mean of $6 \cdot 17$ (range 2-16) minutes, and the sex of the representatives made no significant difference to the length of the visits $(t=1 \cdot 63 ; \mathrm{df}=107$ $\mathrm{p}=0 \cdot 106)$. In each visit a mean of $3.3(1-6)$ products were promoted. Data sheets were offered in 11 of the visits whereas advertising brochures were offered in 51. Two representatives offered copies of articles from peer reviewed journals. I received a gift in 64 visits: these usually displayed an advertisement for the product being promoted, and consisted of stationery, pens, diaries, calendars, or trinkets (mugs, tapes, towels, clothes brush, desk tidy, air freshener, ice scraper, and flower seeds). I was given samples of drugs in 103 of the visits and received 174 samples altogether. The table lists these samples according to the classification used in the British National Formulary.

Free drug samples received by a general practitioner in one year. Drugs classified in accordance with British National Formulary

\begin{tabular}{|c|c|c|}
\hline & $\begin{array}{l}\text { No }(\%) \text { of } \\
\text { samples }\end{array}$ & $\begin{array}{c}\text { Estimated cost } \\
(\mathscr{C})\end{array}$ \\
\hline \multicolumn{3}{|l|}{ Drugs used in treatment of: } \\
\hline Cardiovascular system & $36(21)$ & 376.35 \\
\hline Infections & 29 (17) & 250.94 \\
\hline Respiratory system & 24 (14) & 327.09 \\
\hline Gastrointestinal system & 21 (12) & 155.93 \\
\hline Musculoskeletal and joint diseases & 20 (11) & 147.46 \\
\hline Skin & $12(7)$ & 66.19 \\
\hline Central nervous system & 11 (6) & 47.79 \\
\hline Ear, nose, and oropharynx & (3) & 36.77 \\
\hline Eye & (3) & 14.54 \\
\hline Others & (6) & 62.16 \\
\hline Total & $174(100)$ & 1485.22 \\
\hline
\end{tabular}

\section{Comment}

The code of marketing practice for the pharmaceutical industry states: "The size and quantity of the sample supplied should be appropriate for either familiarisation with the presentation and appearance of the product, or the provision to patients for immediate use of an initial small supply of a product." ${ }_{3}$ The total value of the samples recorded in this study was $£ 1485$, which constitutes a substantial gift to a general practitioner.

Representatives are perceived by the industry as having a dual role as educators and promoters. ${ }^{3}$ During the visits I received, however, the emphasis was on promotion. Advertising has been shown to influence physicians' prescribing habits so that they tend to prescribe analgesics according to advertised guidelines rather than published scientific literature. ${ }^{4}$

The gifts I received were not expensive items. Their main purpose seemed to be an attempt to keep a product's name before me, presumably in the hope that this would be consciously or unconsciously assimilated. Aceptance of gifts can have complex practical and ethical repercussions, including the establishment of a relationship in which a doctor may feel obliged to respond. ${ }^{5}$

\section{I thank Maureen Arrigan for her secretarial help.}

1 Lexchin J. Doctors and detailers: therapeutic education or pharmaceutial promotion? Int $\mathcal{J}$ Health Serv 1989;19:663-79.

2 Smith R. Doctors and the drug industry; too close for comfort. BMf 1986;293:905-6

3 Federation of Irish Chemical Industries. Code of marketing practice for the pharmaceutical industry (third edition, 1984). In: Data sheet compendiun 1991-92. Dublin: FICI, 1991:v-ix.

4 Avorn J, Chen M, Hartley R. Scientific versus commercial sources of influence on the prescribing behaviour of physicians. Am F Med 1982;73:4-8.

Chren MM, I andefeld CS, Murray TH. Doctor, drug companies and gifts. FAMA 1989;262:3448-51

(Accepted 18 April 1993)

\section{A national survey of the provision of support services for people with cancer}

\section{J M Addington-Hall, M W Weir, C Zollman, M B McIllmurray}

As well as technical competence in diagnosis and management people with cancer need psychosocial support. ${ }^{1}$ They may also require help to overcome the physical effects of the disease and of treatment. Although both these needs are increasingly being recognised, little is known about the distribution of services aimed at helping people cope with cancer. This paper describes the results of a national survey of support services provided by district health authorities for people with cancer.

\section{Methods and results}

In January 1991 the district general managers of all 190 English health districts were sent a short postal questionnaire asking about "any rehabilitation or support services provided for patients following treatment or diagnosis of cancer." Four weeks later nonresponders were recontacted.

The response rate was $89 \%$ (170). Six districts said
Correspondence to:

$B M 7$ 1993;306: 1649-50 
Department of

Epidemiology and Public

Health, University College

London, London

WC1E 6EA

J M Addington-Hall, lecturer

in health services research

Wirral Health Authority

M W Weir, director of

community medicine and

quality assurance

Oncology Unit, Bristol

Hospital for Sick Children,

Bristol

C Zollman, senior house

officer

Royal Lancaster Infirmary, Lancaster

M B McIllmurray, consultant physician they provided no relevant services. Sixteen districts provided only practical support (physiotherapy, dietary advice, stoma and mastectomy care), one provided only emotional care (counselling, support groups), and 94 provided both types of support. Fifty three provided some complementary therapies (guided imagery, meditation/relaxation, healing, and art therapy) in addition to these services. The numbers of districts providing each service are listed in the table.

Although 111 districts had informal links with independently run cancer support services, usually with palliative care services (51) or local cancer support groups (45), there was limited evidence that these services were compensating for deficiencies in NHS services: no districts without support services had links with independent organisations; in four of those offering only practical support emotional care was provided outside the NHS; and in one both emotional care and complementary therapies were available from independent organisations.

\section{Comment}

Our results show that there are wide variations in provision of supportive care for patients with cancer. Most districts provided some practical support, usually stoma and mastectomy care. The full range of support, however, was provided in only 32 districts. This may be an underestimation as many hospital dietetics and physiotherapy departments may have provided services for patients with cancer but been overlooked in the completion of the questionnaire because they were not separate specialist cancer services.

Results suggest that the NHS is already responding to the emotional needs of patients with cancer. The availability of counsellors and support groups may, however, be lower than that suggested by our data as we did not ask about the services' nature and accessibility. Some, for example, may have been available only to patients with breast cancer. Some districts at least were referring patients to routinely available clinical psychology or psychiatric services which may not have been available to, or appropriate for, distressed patients with cancer. Macmillan nurses and hospices were often mentioned, suggesting that some services were orientated towards patients in the last stages of their illness.

More districts provided complementary therapies than we had expected. Clearly, the integration of these

Provision of support services for people with cancer by district health authorities

\begin{tabular}{lrc}
\hline & \multicolumn{2}{c}{ Districts reporting provision of service } \\
\cline { 2 - 3 } & No & $\%$ \\
\hline Practical support services & & \\
Specialist physiotherapy & 38 & 22 \\
Dietary advice & 116 & 68 \\
Stoma care & 156 & 92 \\
Mastectomy care & 139 & 82 \\
None of these & 7 & 4 \\
One & 10 & 6 \\
Two & 52 & 31 \\
Three & 69 & 41 \\
Four & 32 & 19 \\
Emotional care & & \\
Counselling & 141 & 83 \\
Support groups & 97 & 57 \\
None of these & 24 & 14 \\
One & 54 & 32 \\
Two & 92 & 54 \\
Complementary therapies & & \\
Guided imagery & 18 & 11 \\
Relaxation-meditation & 41 & 24 \\
Healing & 11 & 11 \\
Art therapy & 18 & 69 \\
None of these & 117 & 15 \\
One & 26 & 16 \\
Two or more & 27 & \\
\hline
\end{tabular}

non-traditional methods into clinical practice has begun, despite the lack of evidence of benefit and of any clear understanding of their role in cancer care.

Our findings suggest that cancer support services are developing in a fragmented and poorly coordinated way. There is no agreed strategy for providing supportive services for patients with cancer and little evidence on which to draw to decide what should be provided and how it should be organised. Further evaluations of models of supportive care $^{3}$ and of specific interventions ${ }^{4}$ are urgently needed. In the meantime we urge those responsible for providing and purchasing services for cancer patients to continue to address the need to provide effective and accessible services aimed at meeting physical and emotional needs.

Smith T Cancer services. BMF 1990;301:1406-7.

2 Watson PG. Cancer rehabilitation: the evolution of a concept. Cancer Nursing 1990;13:2-12.

3 Mcllmurray MB, Gorst DW, Holdcroft PE. A comprehensive service for patients with cancer in a district general hospital. $B M \mathcal{F}$ 1986;292:669-7 4 Fallowfield L, Roberts R. Cancer counselling in the United Kingdom Psychology and Health 1992;6:107-17.

(Accepted 15 April 1993)

\section{Relation between sialic acid concentrations and the haemostatic system in the elderly}

\section{Kazuomi Kario, Takefumi Matsuo}

Department of Internal Medicine, Awaji-Hokutan Public Clinic, Hokutan, Hyogo 656-16, Japan

Kazuomi Kario, physician

\section{Department of Internal} Medicine, Hyogo

Prefectural Awaji Hospital, Sumoto, Hyogo 656, Japan

Takefumi Matsuo, physician

Correspondence to:

Dr K Kario.

BMF 1993;306:1650-1
A recent prospective study showed that mortality due to cardiovascular disease was increased in people with high serum sialic acid concentrations.' Recent reports suggest that smoking and lipid concentrations may be confounders, ${ }^{23}$ but the precise relation between the serum sialic acid concentrations and death from cardiovascular disease remains uncertain. Fibrinogen is a well known risk factor for cardiovascular disease and, like sialic acid, is an acute phase reactant. Plasma fibrinogen concentrations increase with aging and smoking, ${ }^{4}$ characteristics which are similar to those of sialic acid. ${ }^{23}$ The possibility that fibrinogen might be a strong confounder that explains the relation between sialic acid concentrations and cardiovascular death has not been investigated. We therefore investigated the relation between serum sialic acid concentrations and plasma concentrations of various haemostatic variables and the effect of smoking.

\section{Subjects, methods, and results}

We studied 138 healthy Japanese men aged over 60 years (mean age 78 years) who were selected from among men undergoing annual health examinations. Subjects with abnormal results on routine laboratory tests ( $\mathrm{C}$ reactive protein, blood urea nitrogen, creatinine, transaminases, etc) were excluded. Fifty subjects had hypertension and 48 were smokers. Blood samples were collected after an overnight fast, and serum sialic acid concentrations were measured by an enzymatic assay. ${ }^{5}$ Serum total cholesterol and triglyceride concentrations were determined by enzymatic assays using cholesterol esterase, cholesterol oxidase, and glycerol3-phosphate oxidase. The clotting activity of plasma fibrinogen and factor VII was determined by automated one-stage clotting assays. Plasma concentrations of antithrombin III, heparin cofactor II, plasminogen, 